
\title{
\& Research Square \\ An Optimization and Formability Assessment Tool for Incremental Sheet Forming
}

\author{
Maan Tarng Lew \\ Dharmesh Kumar \\ Jirathearanat Suwat \\ Akshay Chaudhari \\ Yunfeng Zhang \\ Senthil Kumar Anantharajan ( $\nabla$ mpeask@nus.edu.sg ) \\ National University of Singapore
}

\section{Research Article}

Keywords: Incremental Sheet Forming (ISF), Formability, Wall Angle, Forming Orientation, Optimization

Posted Date: February 21st, 2022

DOI: https://doi.org/10.21203/rs.3.rs-1323151/v1

License: (1) (i) This work is licensed under a Creative Commons Attribution 4.0 International License.

Read Full License 


\section{Abstract}

Incremental Sheet Forming (ISF) is a novel die-less forming process, which is suitable for small batch production. It has high forming flexibility and is able to form highly complex free form part geometry. However, the parts formed using the ISF process may have geometrical inaccuracies, pillow effect and part failure when the forming wall angle exceeds the limit. Hence, it is important to know the formability of a part ahead in order to plan the ISF process correctly. One method to know the formability is by finding the optimum forming orientation. In the current state of art, some studies were carried out to obtain the optimum forming orientation by using Stereolithography model (STL) on symmetrical parts. However, it is noticed that there is lack of investigations of the optimum forming orientation on nonsymmetrical part. Hence, in this work, a new approach in finding the optimum forming orientation of nonsymmetrical part is proposed by considering the formability of the part along two axes. To implement this new approach, a genetic-algorithm-like method is proposed and explained. The proposed algorithm is integrated with visual color map. The optimized forming orientation, local wall angle, and percentage reduction of sheet thickness are presented in the color map format to further assist the end user. Several experiments were conducted to test the proposed algorithm. From the experimental investigations, it can be concluded that the proposed algorithm helps the user to identify potential failure areas and, thus, reduce the failures by reorienting the part forming direction.

\section{Introduction}

Incremental Sheet Forming (ISF) is a flexible and low-cost process die-less forming process, which is suitable for forming user-defined geometries in a small batch production environment. In ISF forming process, a sheet metal blank clamped between the bottom backing plate and top blank holders is formed incrementally by a specialized forming tool [1]. The forming tool motion is controlled by the Computer Numerical Controlled (CNC) codes, which are generated using a Computer Aided Manufacturing (CAM) software. This tool motion is known as forming tool path. In general the tool moves down in a step-down manner as shown in Fig. 1 where a geometry with wall angle (90-a) is being formed.

Although ISF has its flexibility in forming various part geometries, it has some drawbacks such as part failure and geometrical inaccuracy. One of the methods to improve the part geometrical inaccuracy is by finding the optimum forming orientation of a part. Zhu et.al [2] carried out the optimum forming orientation of cone geometry by using the Stereolithography (STL) model. The optimum forming direction was obtained by evaluating the formability of the cone at one forming axis. It was found that by using the optimum forming orientation, where a part was formed in a different forming posture, and the part thickness was more evenly distributed. Besides that, Zhu et.al [3] also investigated the formability of a part by titling the forming tool at different direction. It was found that by tilting the forming tool at a given direction, the part thickness distribution could be more uniformly distributed. Figure 2 shows the different forming postures of a CAD model. 
Besides the forming orientation, the forming wall angle limit is also an important factor in determining the formability of a part. As pointed out by Jesweit et. al [4], the formability of ISF for different materials at a given thickness can be quantified using maximum draw angle, or maximum forming wall angle. For instance, the maximum forming wall angle for a $1 \mathrm{~mm}$-thick brass is $40^{\circ}$. Once the forming wall angle exceeds the maximum forming wall angle, part failure may happen. Table 1 shows the maximum forming wall angle for different materials at a given thickness. It is known that the predicted final sheet thickness of a formable part is governed by the sine law[5], $t_{f}=t_{j} \sin (90-\phi)$, where $t_{f}$ is the final sheet thickness, $t_{i}$ is the initial sheet thickness, and $\phi$ is the wall angle. On the other hand, Yang et.al [6] showed that the sheet thickness thinning ratio is also an indicating factor for the formability in ISF.

Table 1

Maximum forming wall angle for different material at given thickness [4]

\begin{tabular}{|lll|}
\hline Material & $\varphi_{\max }$ (maximum forming wall angle) & $\mathrm{t}_{\mathrm{o}}, \mathrm{mm}$ (initial thickness) \\
\hline AA 1050-0 & $67.5^{\circ}$ & 1.21 \\
\hline AA 6114-T4 & $60^{\circ}$ & 1.0 \\
\hline Al 3003 - O & $78.1^{\circ}$ & 2.1 \\
\cline { 2 - 3 } & $72.1^{\circ}$ & 1.3 \\
\cline { 2 - 3 } & $71^{\circ}$ & 1.21 \\
\hline Al 5754 - O & $67^{\circ}$ & 0.93 \\
\hline Al 5182 - O & $62^{\circ}$ & 1.02 \\
\hline AA 6111-T4P & $63^{\circ}$ & 0.93 \\
\hline DC04, mild steel & $53^{\circ}$ & 0.93 \\
\hline DDQ (Deep Drawing Quality) & $70^{\circ}$ & 1.0 \\
\hline HSS (High Speed Steel) & $65^{\circ}$ & 1.0 \\
\hline Copper & $65^{\circ}$ & 1.0 \\
\hline Brass & $40^{\circ}$ & 1.0 \\
\hline
\end{tabular}

Although different studies had been carried out on forming orientation, it is noticed that there is lack of investigations on the optimum forming orientation of non-symmetrical part. It is noticed that in the proposed approach [2, 3], the formability of a part was evaluated along a single axis, which may be not suitable for non-symmetrical part. Hence, in this work, a new approach to find the optimum forming 
orientation of non-symmetrical part is proposed by considering the formability along two axes. In the optimization process, a genetic-algorithm-like approach is developed and implemented. In addition, the proposed algorithm is integrated with visual color map of a formed part. The optimized forming orientation, local wall angle, and percentage reduction of sheet thickness are presented in a color map format to facilitate the end users. Several experiments are conducted to validate the effectiveness of the proposed algorithm.

\section{Development Of Optimization And Formability Assessment Algorithm}

In this section, the development of the algorithm is discussed in detail. There are two distinct activities; viz, optimized forming orientation, formability assessment using color map of wall angle and sheet thickness reduction. The detail of the development process for each algorithm is also presented.

\subsection{Overview of the Proposed Algorithm}

In this proposed algorithm, the required CAD model to be formed is given as an input in STL(ASCII) format, where the model is represented using discretized triangles and normal vectors [7-9]. First, the optimum forming orientation of a CAD model is found by evaluating the formability at two axes. To obtain this optimized orientation, designated evaluation criteria are chosen: forming angle limit, and critical area which is the total triangle area in a CAD model in which its angle is more than the forming angle limit. During the evaluation process, first the algorithm will check all the angles in the STL file are less than forming angle limit. If this criterion is being satisfied, the evaluation process will stop here, and the same evaluation process will be carried out for same CAD model in different orientation. Otherwise, the algorithm will evaluate the critical area of the CAD model by performing the summation of the area in a CAD model in which its angle is more than the angle limit.

Second, the CAD model is analyzed using the color map, to visually inspect at the potential forming errors in the CAD model, such as high forming wall angle or high thickness reduction, and decide whether or not to form the part using ISF. It is suggested to redesign the CAD model if high wall angle or high thickness reduction is seen from the color map.

The following steps summarize the overview function and working principle of the proposed algorithm.

1. Get the CAD model in STL (ASCII) format.

2. Perform the optimization of forming orientation.

3. Perform the color map plot of the CAD model for wall angle and thickness reduction.

4. Evaluate the color plot and make decision. If the wall angle and the thickness reduction are acceptable, user can proceed the ISF forming. Otherwise, user is advised to redesign the CAD model. 


\section{Perform the ISF forming.}

The algorithm for getting the optimized forming orientation of a CAD model, and color map of wall angle, color map of sheet thickness reduction, and will be discussed in the next section.

\subsection{Optimized Forming Orientation Algorithm}

Figure 3a shows a CAD model with forming direction in $\mathrm{Y}$-axis and the possible rotation combination along two axes. This $C A D$ model can be rotated along $X$ and $Z$ axes, and the angle of these rotations are represented by $\theta_{x}$ and $\theta_{z}$. Figure $3 \mathrm{~b}$ shows the sample of CAD models with and without rotation along two axes. The optimized forming direction of this CAD model is an arbitrary combination of rotation along $X$ and $Z$ axes, which is currently unknown. The objective function and constraint for the optimization are given in Eq. 1 and Eq. 2. Here, the design variables are $\theta_{X}$ and $\theta_{z}$ which represent the angle of rotation along $X$ and $Z$ axes.

$$
\begin{gathered}
\min f\left(\theta_{X^{\prime}} \theta_{Z}\right)(E q .1) \\
\text { s. } t\left(\theta_{X^{\prime}} \theta_{Z}\right) \leq 90 \text { and }\left(\theta_{X^{\prime}} \theta_{Z}\right) \geq 0 \text { (Eq.2) }
\end{gathered}
$$

Since a repetitive search operation has to be involved to solve this optimization problem, a geneticalgorithm-like approach is chosen in this paper. This approach has two similar steps in normal genetic algorithm, which are repetitive of initialization of a population, selection based on fitness function.

The first step in this genetic-algorithm-like approach is initialization of population, which is illustrated in Fig. 4. There are four divisions in the design space, namely AC-AC, $A C-C, C-A C$, and $C-C$, which are the possible region to find the optimized forming orientation of a CAD model. $A C$ is the acronym for anticlockwise rotation, and $C$ for clockwise rotation. In each division region, it will be populated with different $C A D$ model at various combination of rotation along two axes ( $X$ and $Z$ ). For example, in the AC-AC region, all the $C A D$ models will be at various combination of rotation along $X$ and $Z$ axes with all anticlockwise manner. The coordinate transform formula, which is shown in Eq. 3, is used to perform rotation on model coordinate. To generate a new CAD model, the window file stream has to be used to write the newly rotated coordinates with normal vector into a new file.

$$
\left(\begin{array}{l}
x^{\prime} \\
y^{\prime} \\
z^{\prime}
\end{array}\right)=\left(\begin{array}{ccc}
\cos \theta & \sin \theta & 0 \\
-\sin \theta & \cos \theta & 0 \\
0 & 0 & 1
\end{array}\right)\left(\begin{array}{l}
x \\
y \\
z
\end{array}\right), x y z=\text { oldcoordinate, } x^{\prime} y^{\prime} z^{\prime}=\operatorname{newcoordinate}(E q .3)
$$

The following steps summarizes the function to rotate and generate a new CAD model:

1. Get a CAD model in STL (ASCII) format. 
2. Use file stream to read the STL file.

3. Store all the current coordinate into a list or dictionary using a loop.

4. Get the rotation values from user.

5. Apply the coordinate transformation at X-axis and Z-axis to the coordinates.

6. Calculate the new normal vectors from the new coordinates using cross product.

7. Use file stream to write a new file in STL format with all the new coordinates and normal vectors. The second step is to build the selection criteria to evaluate the fitness of a population based on two criteria. The first evaluation criteria is to calculate how much all the wall angle in a CAD model different from the forming angle limit. However, not all CAD models can fulfill this first evaluation criteria as it is difficult to be achieved in some complex geometries, such as freeform shape. Hence, a second evaluation criteria is required, which is the evaluation of critical area, which is the summation of the triangle area in a CAD model which more than its forming angle limit. To calculate the triangle area, Eq. 4 is used, where $\overrightarrow{A B}$ and $\overrightarrow{A C}$ are the vectors of a triangle.

area of a triangle $=0.5 *(|\overrightarrow{A B} x \overrightarrow{A C}|)$ (Eq.4)

The following steps summarizes the selection criteria:

1. Get a CAD model in STL (ASCII) format.

2. Evaluate the fitness using first criteria, where it calculates how much all the wall angle in a CAD model different from the forming angle limit.

3. If there is wall angle in CAD model which is more than the forming angle limit, then use the second evaluation criteria.

Once the selection criteria are confirmed, the overall optimization process is carried out by iterative searching, which is illustrated in Fig. 5. The search approach is divided into two portions: coarse search and fine search. The purpose of coarse search is to get the searching direction and reduce the searching time. The fit population in the respective region in design space is identified through coarse search. After that, the fine search operation is carried out by populating the fit region with more population, and evaluating the population to find the optimum result.

The following steps summarize this genetic-algorithm-like search approach:

1. Make sure the input CAD model is in STL (ASCII) format.

2. Perform the coarse search to identify the search direction or region.

3. In the coarse search, perform the first evaluation. If this criteria is met, proceed to fine search with the same evaluation criteria.

4. In the coarse search, if the first criteria is not met, use the second criteria for evaluation.

5. Once the fit region is identified, perform the fine search using the same evaluation criteria.

6. The optimized orientation is the orientation in which a CAD model has the minimum critical area. 


\subsection{Formability Assessment Algorithm Using Visual Color Map}

The purpose of having color map of a CAD model using wall angle and thickness reduction is to give an intuitive assessment to user about the current CAD design. To authors' best knowledge, there is no available CAD software which can perform the mentioned color map of a CAD model.

The normal vectors in STL file is represented in Eq. 5, and the wall angle in respective forming direction can be obtained using Eq. 6-Eq. 8. On the other hand, the thickness reduction can be calculated from Sine rule, which is shown in Eq. 9-Eq. 10.

$$
\begin{gathered}
\vec{n}=n_{x}+n_{y}+n_{z}(E q .5) \\
\theta_{x-\text { formingdirection }}=\cos ^{-1}\left(n_{x}\right) \frac{180}{\Pi}(E q .6) \\
\theta_{y-\text { formingdirection }}=\cos ^{-1}\left(n_{y}\right) \frac{180}{\Pi}(E q .7) \\
\theta_{z-\text { formingdirection }}=\cos ^{-1}\left(n_{z}\right) \frac{180}{\Pi}(\text { Eq.8) } \\
\text { thicknessreduction }=\frac{t_{i}-t_{f}}{t_{i}}, t_{i}=\text { initialthickness, } t_{f}=\text { finalthickness(Eq.9) } \\
t_{f}=t_{i} * \sin (90-\theta)(\text { Eq.10) }
\end{gathered}
$$

To plot the STL file, a proper computer graphic library is required. There are some open source libraries, such as OpenGL, DirectX, and so on. In this paper, OpenTK, which is a C\# version of OpenGL is chosen. For the first division range in color map, it is set to change for every 5-degree, starting from the lowest angle value $\left(0^{\circ}\right)$ to $30^{\circ}$. For the second division range in color map, it is set to the ratio of angle difference (wall angle limit- $30^{\circ}$ ) to 5 . The following steps summarizes the procedures to set the division range in color map:

1. For lowest wall angles $\left(0^{\circ}\right)$, the color is green.

2. For wall angles which are higher or equal to angle limit, the color is red.

3. For the color in between first division range, change the color from green to yellow for every $5^{\circ}$ difference.

4. For the color in between second division range, change the color from yellow to red using the ratio of angle difference (wall angle limit-30 $0^{\circ}$ ) to 5 .

Once the color map procedure is ready, a query function is required to facilitate user in checking the forming wall angle and thickness reduction of a CAD model. To create this function, the selection mode in 
OpenTK has to be used instead of the normal render mode to keep track and store the model coordinates, wall angle, and thickness reduction.

The following steps summarized the proposed color map algorithm for wall angle and thickness reduction:

1. Prepare the forming CAD model and make sure it is in STL (ASCII) format.

2. Read the STL file using file stream and choose a data structure. In this case, dictionary is used.

3. Store the normal vector and coordinates of each triangle of a STL file into dictionary.

4. For color map of wall angle, the angle of each triangle in STL file has to be additionally stored into dictionary.

5. For color map of thickness reduction, the thickness reduction of each triangle in STL file has to be additionally stored into dictionary.

6. To show the color map of wall angle, loop through the dictionary and use the plot function in OpenTK in the render mode. The same step is used for color map of thickness reduction.

7. To implement the query function, use the select mode in OpenTK to keep track the wall angle and thickness reduction.

8. User can assess the formability of a CAD model now by using the wall angle and thickness reduction.

\section{The Developed Graphical User Interface (Gui)}

The proposed algorithm is encapsulated into a Graphical User Interface (GUI) by using C\# language. There are three tabs in the GUI for the color map of wall angle, sheet thickness reduction, and optimized forming orientation. Figure 6 shows the developed GUI with the required input fields from user, such as the STL file path, forming angle limit, and forming direction.

\section{Case Studies And Discussions}

\section{Sample result from the proposed algorithm:}

Figure 7 shows the original and optimized orientation of simple and complex CAD model. The critical area, which is indicated as red color, is almost vanished in the CAD model with optimized orientation. Figure 8 shows the wall angle query function from the developed algorithm.

\section{Experimental Methods:}

The incremental sheet forming process was carried out on annealed Aluminum 2024 and 6061 material of sizes $335 \mathrm{~mm} \times 335 \mathrm{~mm}$ with $1.0-2.0 \mathrm{~mm}$ thickness. During the forming experiments, backing plate on the bottom side and blank holder to hold the blank on top side through bolts were used in the CNC machine center and with robot end-effector. The ISF forming setup using robot end-effector is shown in Fig. 9. The SKD 11 forming tool of $15 \mathrm{~mm}$ diameter with hemi-spherical tip was used to press the blank. 
CCD-200 lubricant was used on the forming surface to reduce the friction between tool and blank. The tool feed rate, depth of cut was $2000-3000 \mathrm{~mm} / \mathrm{min}$, and $0.2 \mathrm{~mm}$ respectively. Powermill Ultimate 2020 software from Autodesk $\AA$ was utilized to generate the spiral pattern tool path for the truncated pyramid geometry and multi-curvature part geometry. Later, thickness values of formed parts were measured using ultrasonic probe and the part wall angles were measured using SolidWorks software for analysis.

\subsection{Case Studies}

Fundamental geometries: A comparison of wall thinning prediction using software and actual formed part thinning for various symmetric and asymmetric geometries is conducted. Initially, some fundamental symmetric geometrical part features such as flat (55deg. wall angle), convex (55-20deg.) and concave (20-55 deg.) are considered. It is observed that part thinning estimated by software based on Sine rule agrees well with the experimental measurements as illustrated in Fig. 10. For example, experimental maximum thinning is $45 \%, 34 \%, 41 \%$ for flat, convex and concave wall respectively, which is similar to thickness reduction estimated by software as illustrated in colored images and legends. Similarly, as shown in Fig. 11 the wall angle predicted by software is matching with the actual wall angles obtained from the CAD geometry. The material formed in this case was Al2024-0 of $1 \mathrm{~mm}$ thickness with $15 \mathrm{~mm}$ SKD11 tool, $2000 \mathrm{~mm} / \mathrm{min}$ feed rate, $0.2 \mathrm{~mm}$ depth of cut and $130 \mathrm{rpm}$ spindle speed at CNC centre.

Complex Geometries: A complex multi-curvature geometry from personalised racing car seat is considered for further investigation. This part is formed using the optimized orientation using the developed algorithm. The red region showed in the color map is due to the addendum design. From Fig. 12 , it can be noticed that maximum thinning is $49 \%$ from forming experiments and algorithm estimated the thinning in the same range $(\sim 50 \%)$. It is also concluded that if the wall angle on the forming wall is less than $20 \mathrm{deg}$. then it is likely to develop a pillow effect within the formed part. In Fig. 12(b), a centre region with wall angle less than $20 \mathrm{deg}$. represented by green colour shows pillow effect on actual formed part (refer highlighted section in Fig. 12(c). The material formed in this case was Al6061-0 of $1 \mathrm{~mm}$ thickness with $15 \mathrm{~mm}$ SKD 11 tool, $3000 \mathrm{~mm} / \mathrm{min}$ feed rate, $0.2 \mathrm{~mm}$ depth of cut using ABB robot endeffector.

However, it should be noted that this software is developed based on the algorithm which follows Sine rule for thinning estimation. Hence, it does not account for the material behavior and forming process conditions.

Analysis of Fractured parts: truncated pyramid geometrical part feature of wall angle 65 deg. was considered here for $1 \mathrm{~mm}$ Aluminum material. Part fractured in the initial stage of the forming and thinning was close to $50 \%$ as estimated by the software, as shown in Fig. 13.

\section{Conclusions}

A new approach to determine optimized forming orientation of non-symmetrical parts was introduced. The detailed process of the optimization and implementation using a genetic-algorithm-like method were 
presented. This proposed algorithm was integrated into a Graphical User Interface (GUI), together with other useful tools, such as wall angle and sheet thickness reduction color map. To substantiate this study, few experimental case studies were carried out to verify the effectiveness of the developed algorithm. From the experimental studies, it was found that this developed tool is able to generate optimized forming orientation, intuitive color maps of wall angle and sheet thickness reduction, which were in good agreement with experiments. This developed optimization and formability assessment tool sets and software can be useful for industry adoption of this incremental sheet forming technology.

\section{Declarations}

\section{Funding}

The study is supported by A-STAR, Singapore, funding scheme RIE2020 Advanced Manufacturing and Engineering (AME) IAF-PP, grant number A1896a0034.

\section{Competing Interests}

The authors declare that they have no financial interests.

\section{Authors Contributions}

All authors contributed to the study conception and design. Material preparation, data collection and analysis were performed by Lew Maan Tarng, Dharmesh Kumar and Akshay Chaudhari. The first draft of the manuscript was written by Lew Maan Trang and all authors commented on previous versions of the manuscript. All authors read and approved the final manuscript.

\section{References}

1. Tisza M (2012) General overview of sheet incremental forming. J achievements Mater Manuf Eng 55(1):113-120

2. Hu Z, Jin J, Jinlan B (2017) Research on the forming direction optimization for the uniformity of the sheet part thickness in the CNC incremental forming. Int J Adv Manuf Technol 93(5):2547-2559

3. Zhu H, Han F, Liu Y (2018) The effect of the extrusion direction on the incremental forming quality considering tool deformation. Int J Adv Manuf Technol 97(5):1835-1846

4. Jeswiet $J$ et al (2005) Asymmetric single point incremental forming of sheet metal. CIRP Ann 54(2):88-114

5. Gatea S, Ou H, McCartney G (2016) Review on the influence of process parameters in incremental sheet forming. Int J Adv Manuf Technol 87(1):479-499

6. Yang $\mathrm{M}$ et al (2018) Study on thickness thinning ratio of the forming parts in single point incremental forming process. Advances in Materials Science and Engineering, 2018

7. Szilvśi-Nagy M, Matyasi GY (2003) Analysis of STL files. Math Comput Model 38(7-9):945-960 
8. Eragubi M (2013) Slicing 3D CAD model in STL format and laser path generation. Int J Innov Manage Technol 4(4):410

9. Adnan FA, Romlay FRM, Shafiq M (2018) Real-time slicing algorithm for Stereolithography (STL) CAD model applied in additive manufacturing industry. in IOP conference series: materials science and engineering. IOP Publishing

\section{Figures}
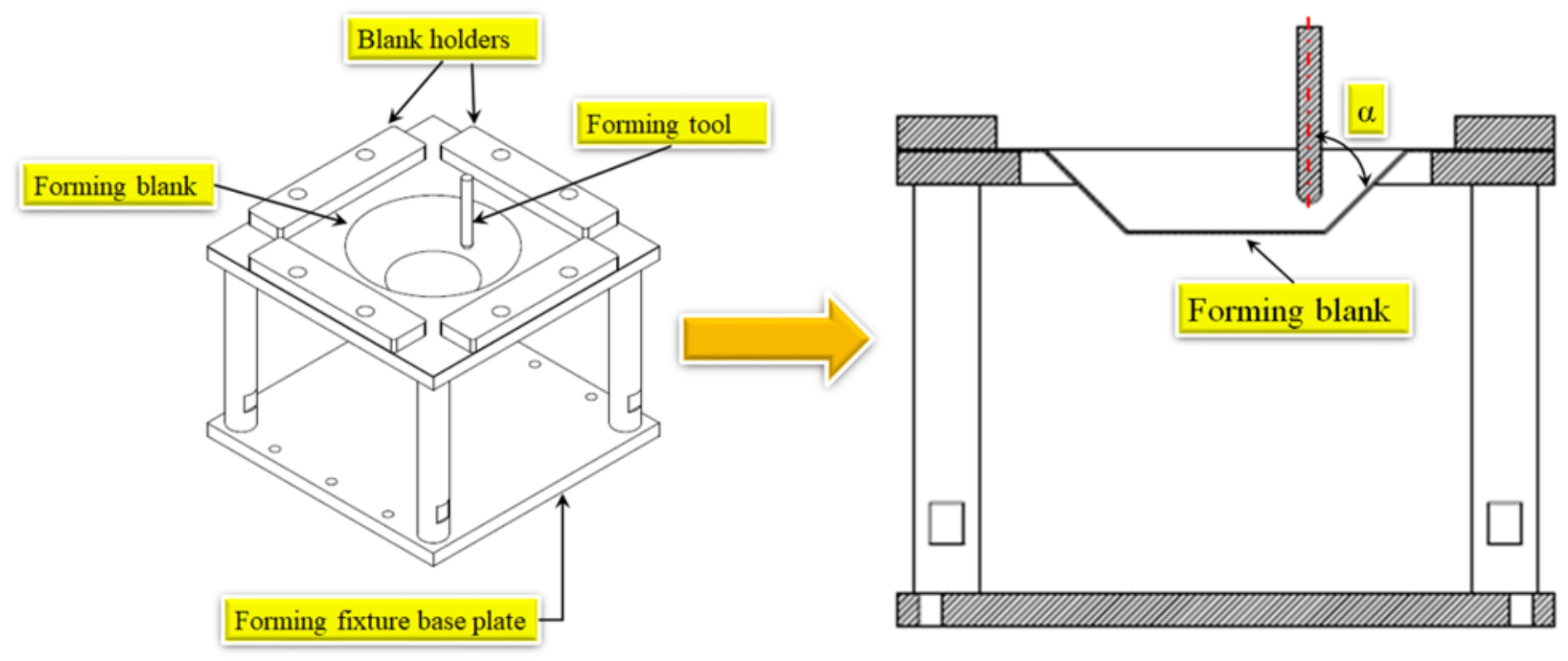

\section{Figure 1}

Setup of ISF process 


\section{Different forming postures}

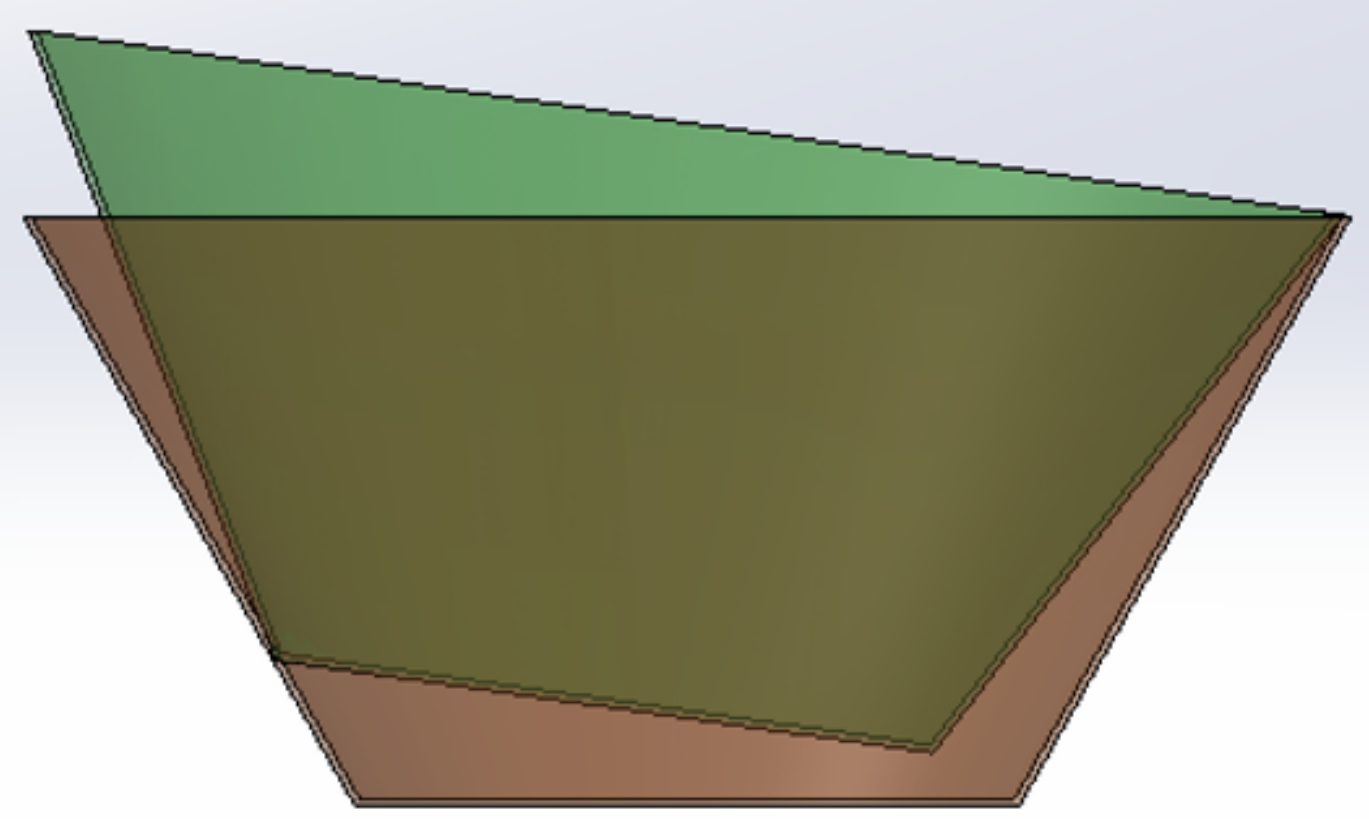

Figure 2

\section{Different forming postures}

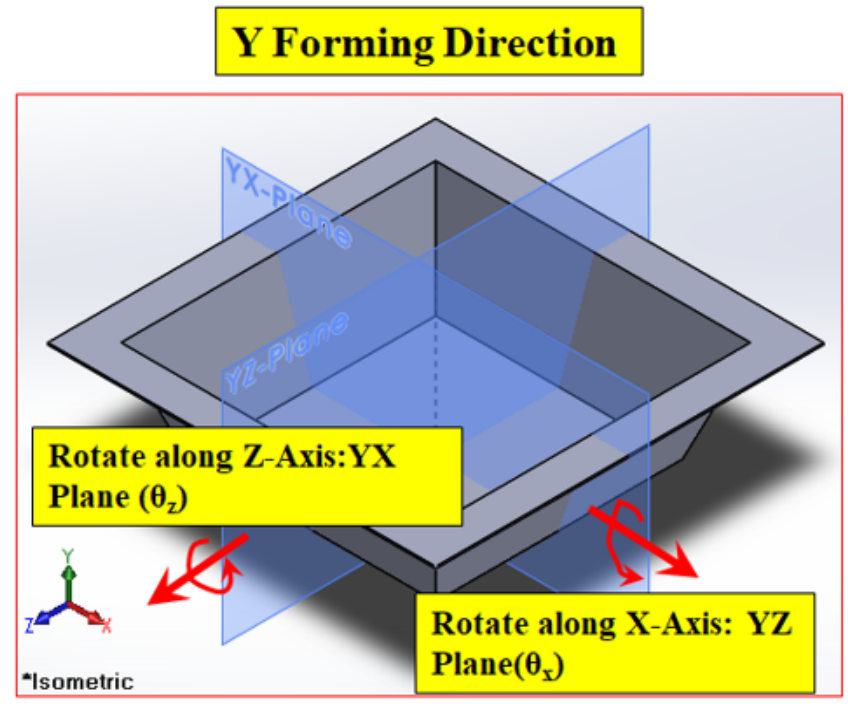

a

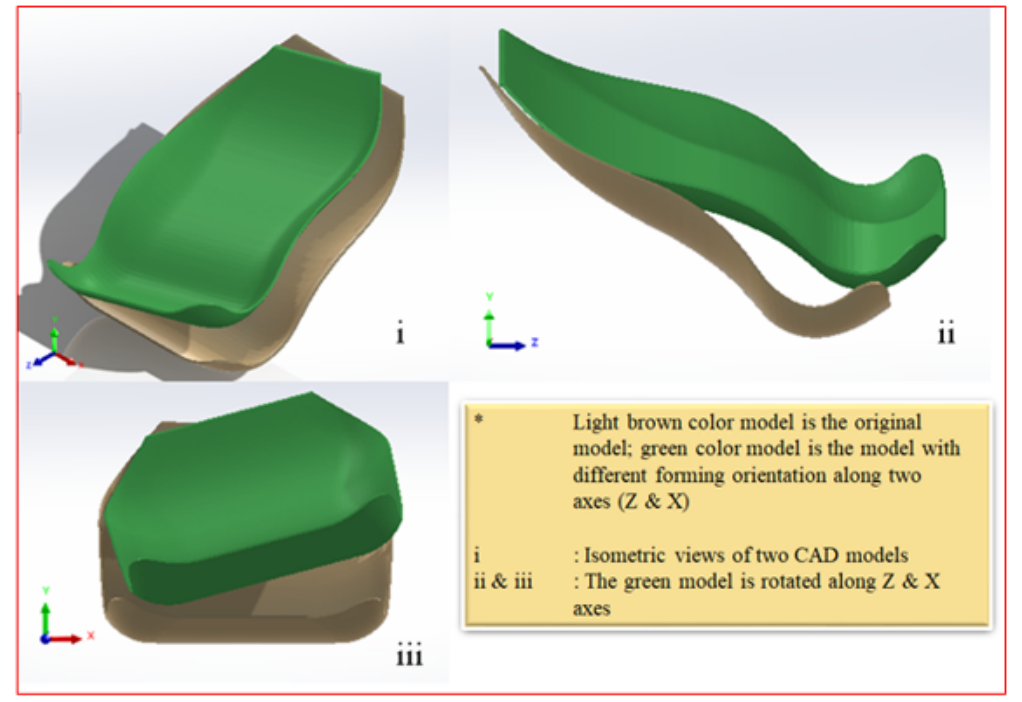

b

Figure 3 
(a) The possible rotation combination along two axes, (b) sample of CAD models with and without rotation along two axes

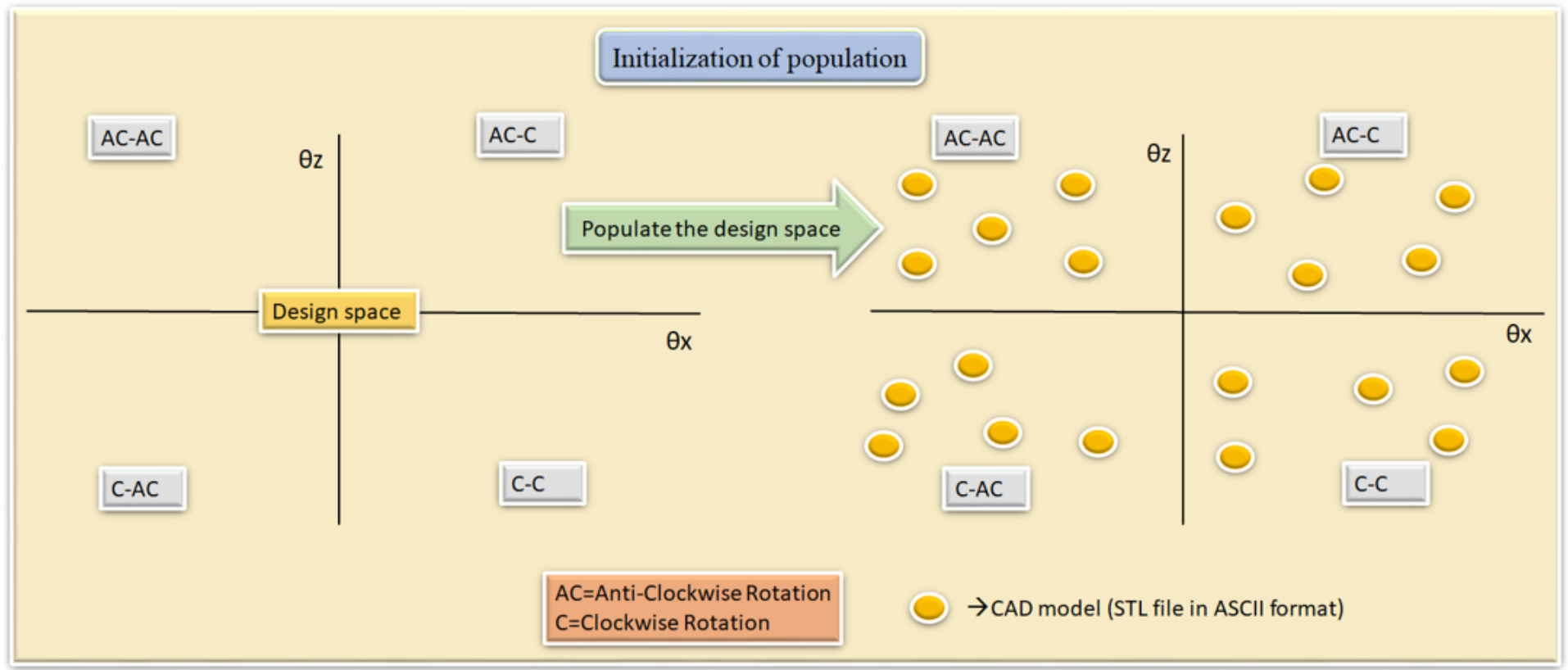

\section{Figure 4}

Initialization of population

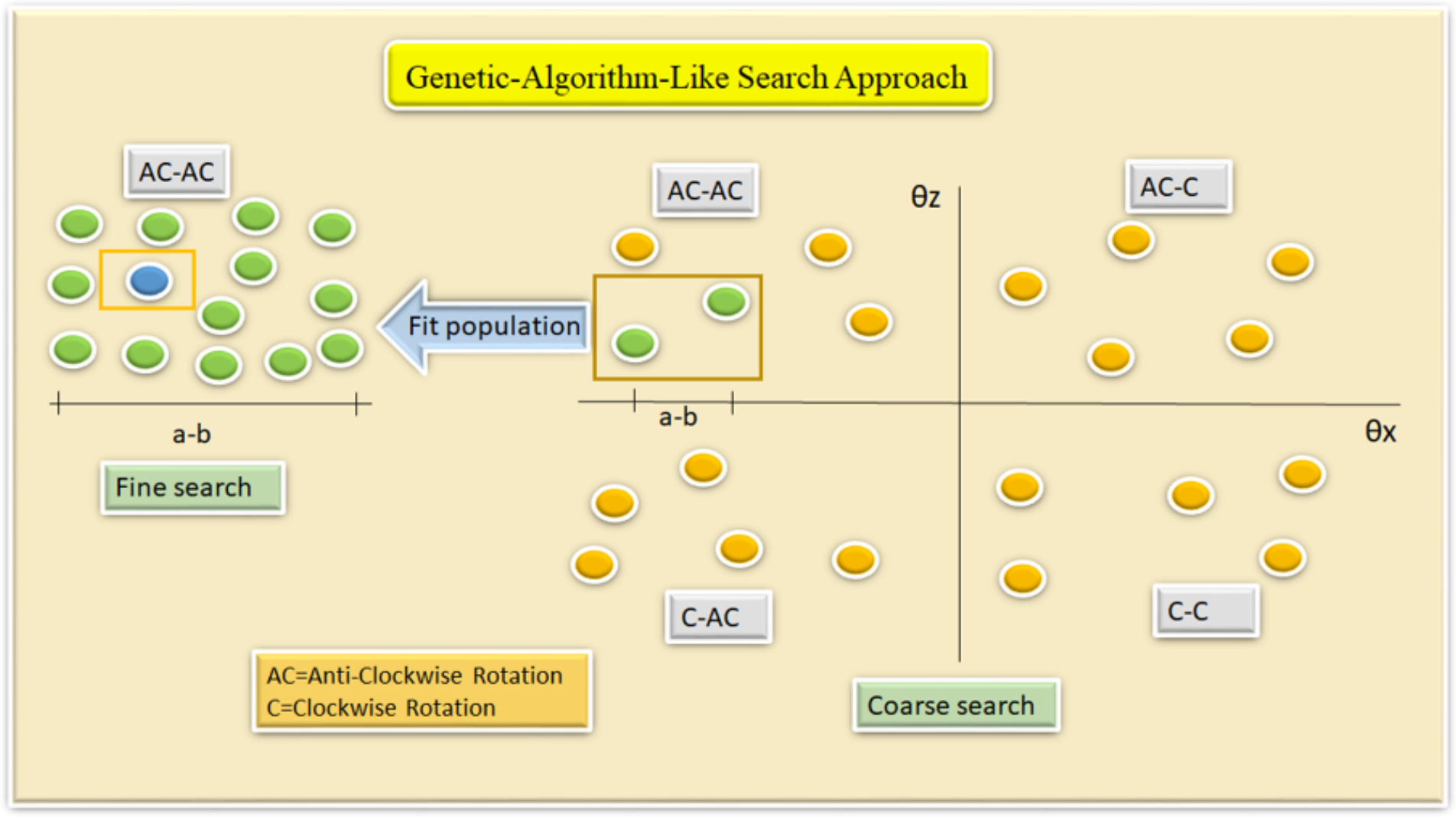

Figure 5 
ISF3P $(1.0 .0)$

Wall Angle Color Map Thickness Reduction Color Map Forming Direction Optimization

File

STL (ASCII) File Path:

Wall Angle Limit (Degree):

Select the correct forming direction:
$\mathrm{X}$
$\mathrm{Y}$
$\mathrm{Z}$
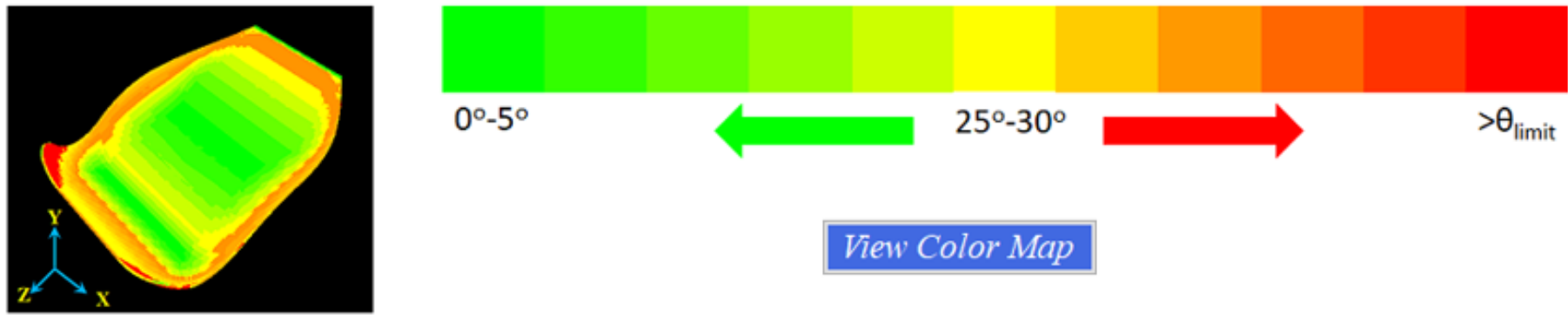

View Color Map

Figure 6

The developed GUI- wall angle color map tab

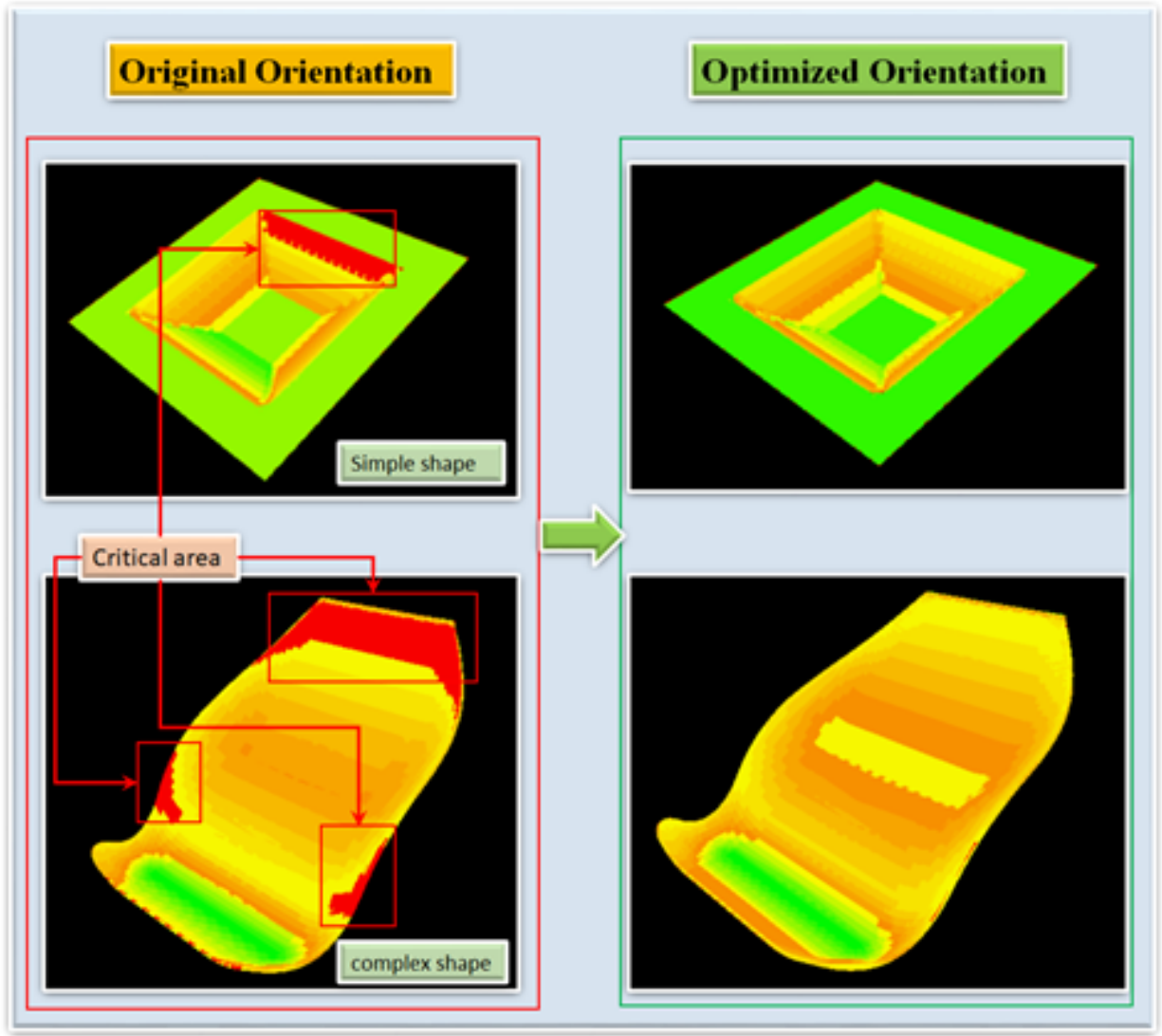

Page $14 / 20$ 
Figure 7

The original and optimized orientation

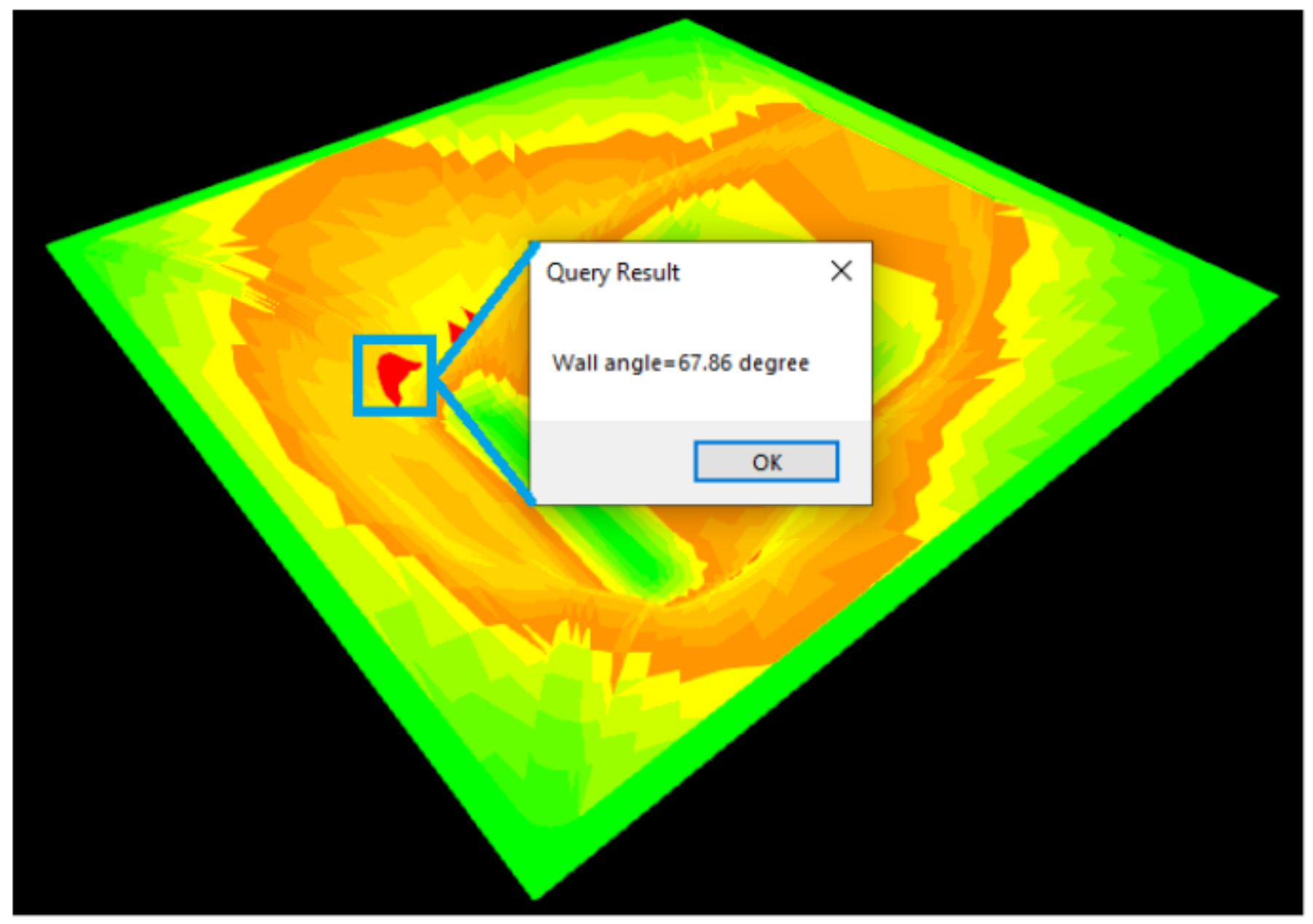

Figure 8

The wall angle query function 


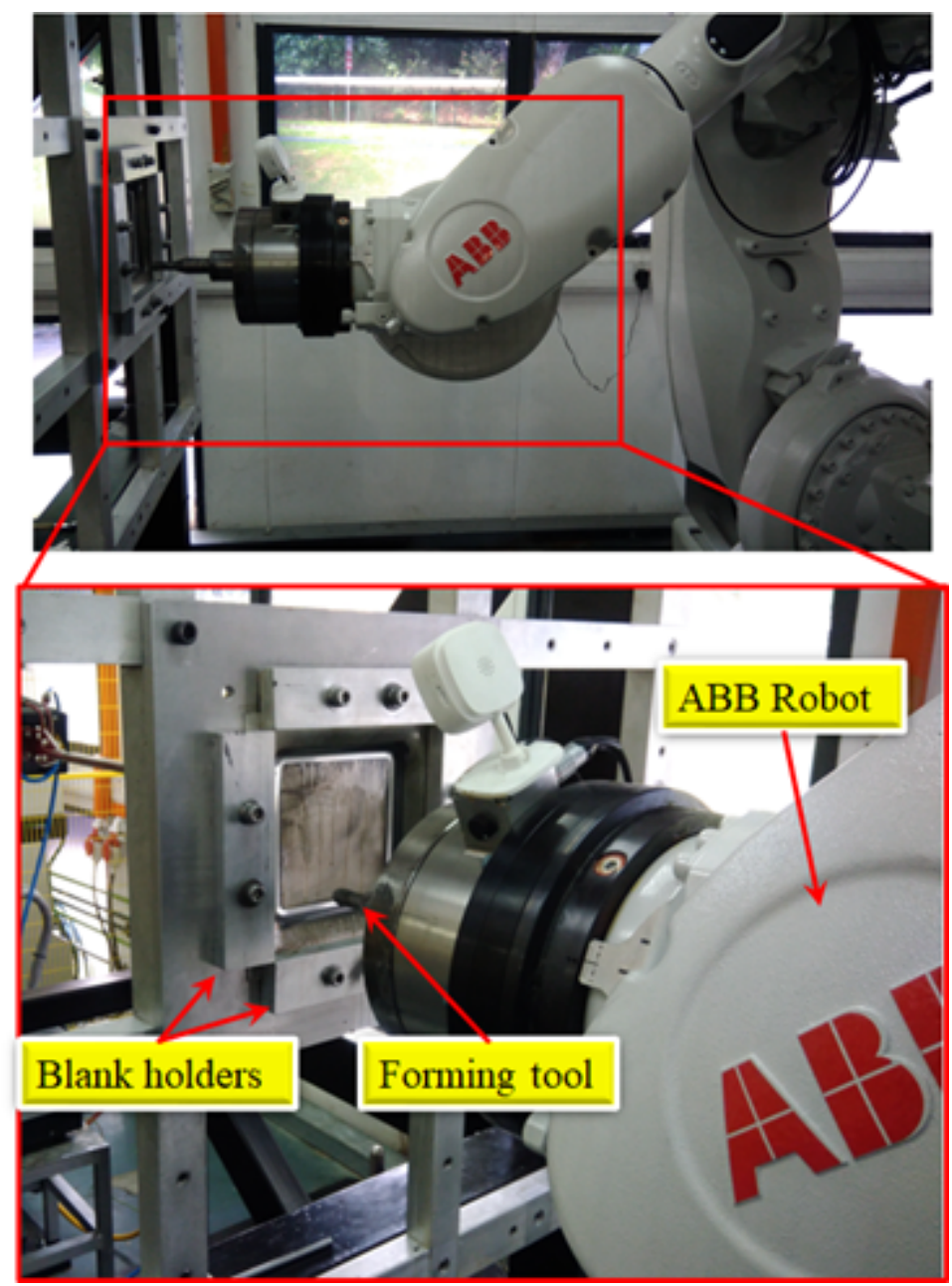

Figure 9

ISF forming setup with robot end-effector 


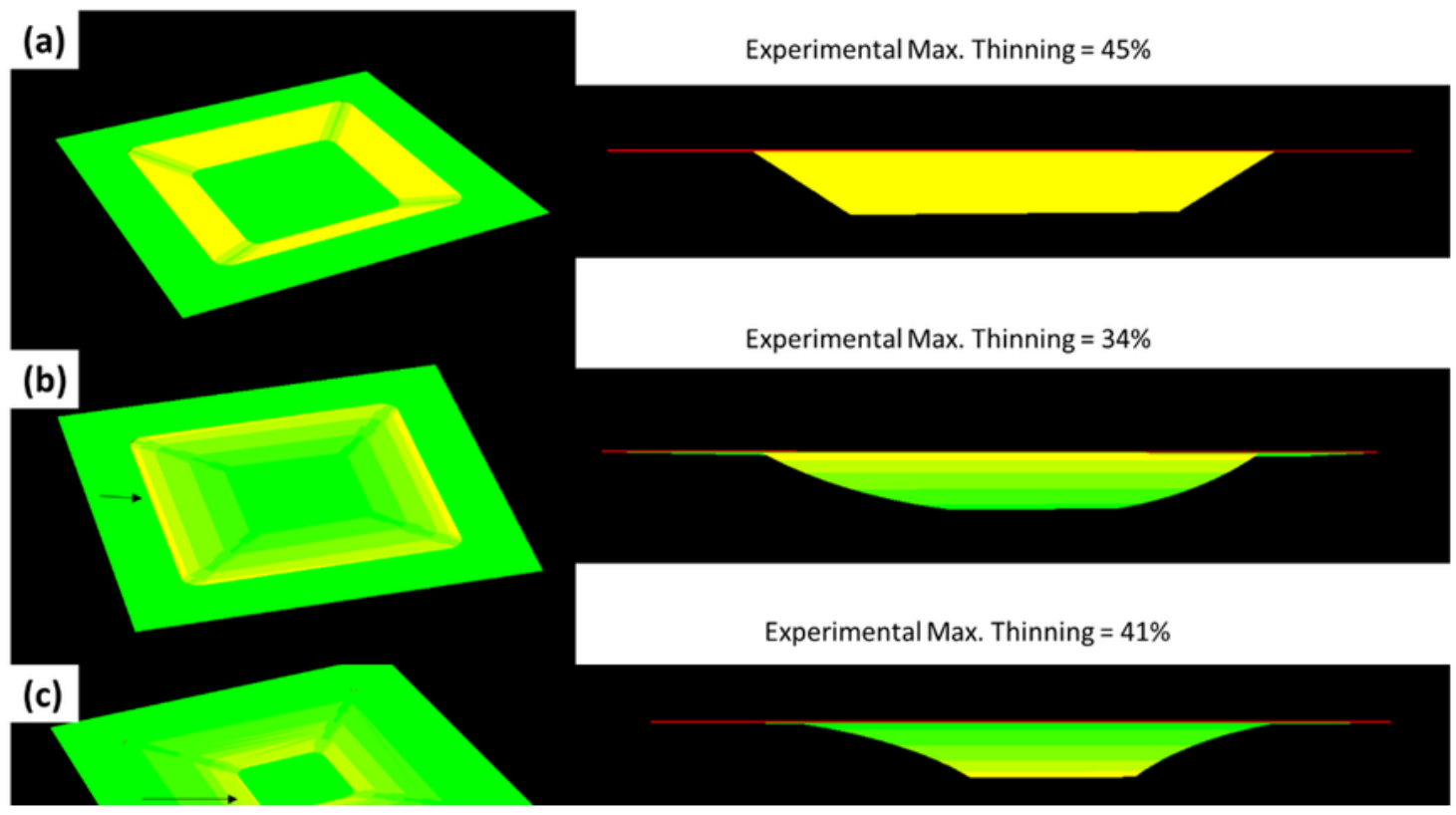

Figure 10

Part thinning for (a) flat (b) convex (c) concave using software, actual formed part of (d) flat (e) convex (f) concave geometry 


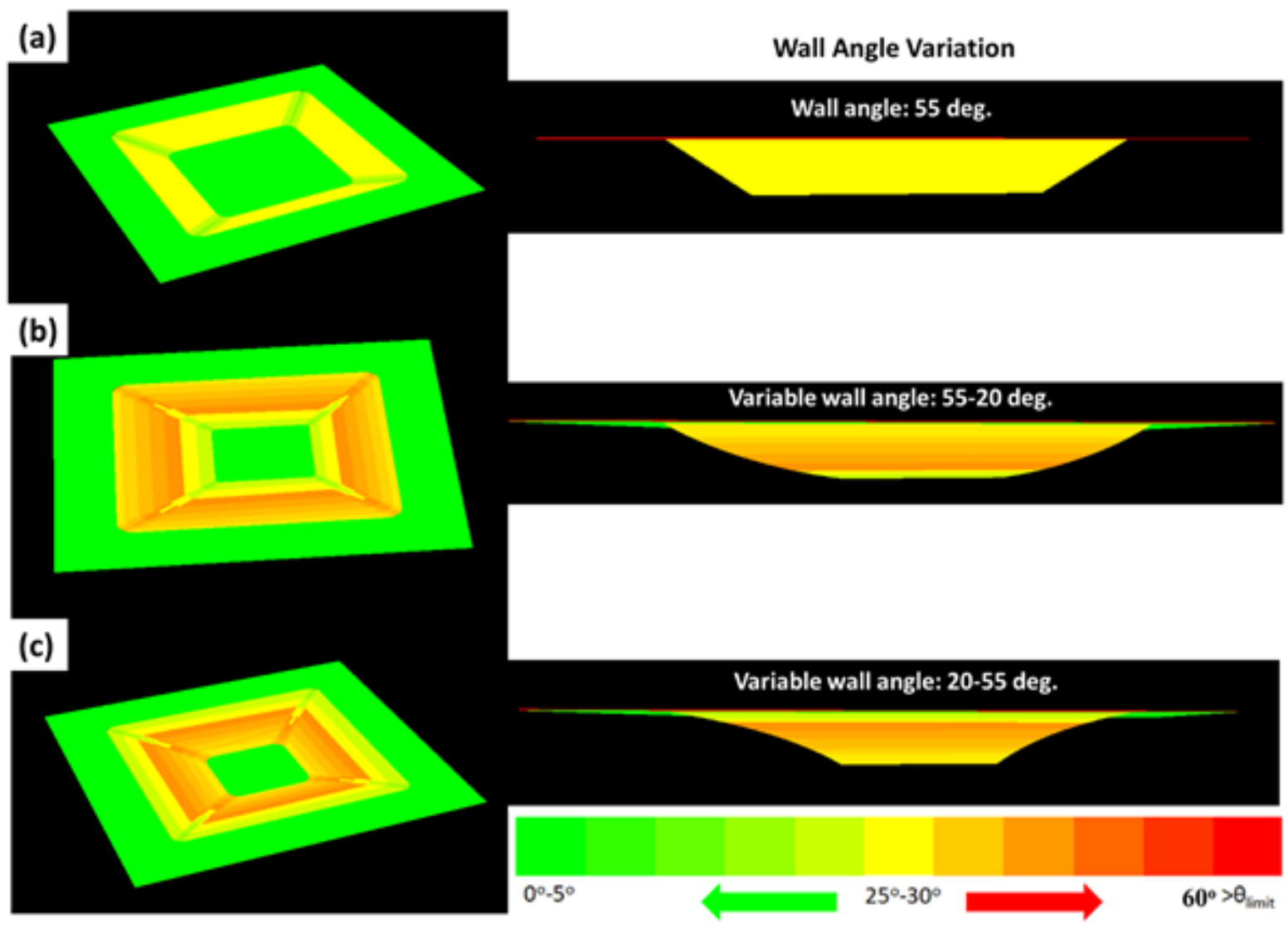

Figure 11

Wall angle variation for (a) flat (b) convex (c) concave geometry 

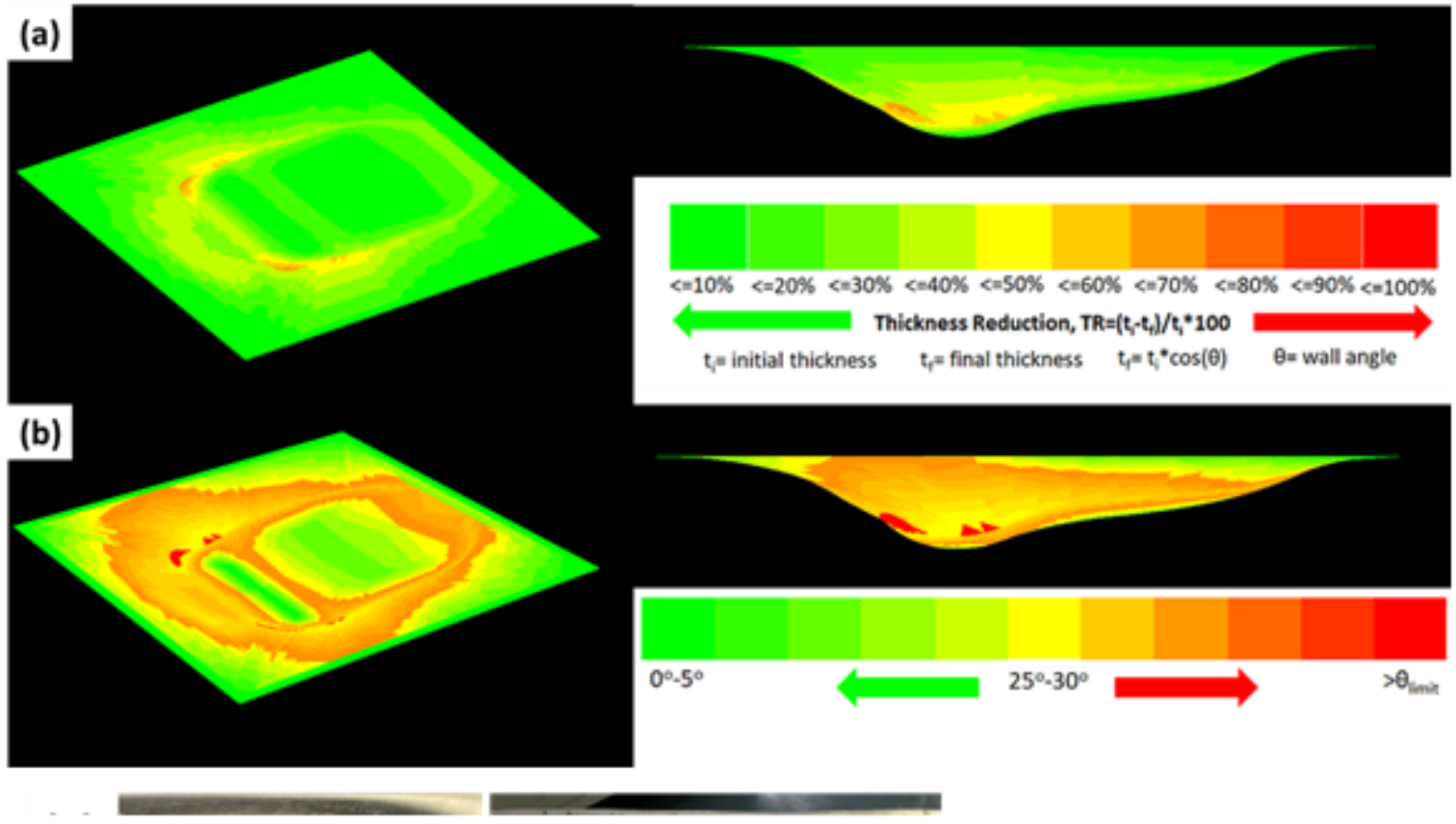

Figure 12

Racing car seat (a) Part thinning (b) wall angle estimation using software (c) actual formed part 


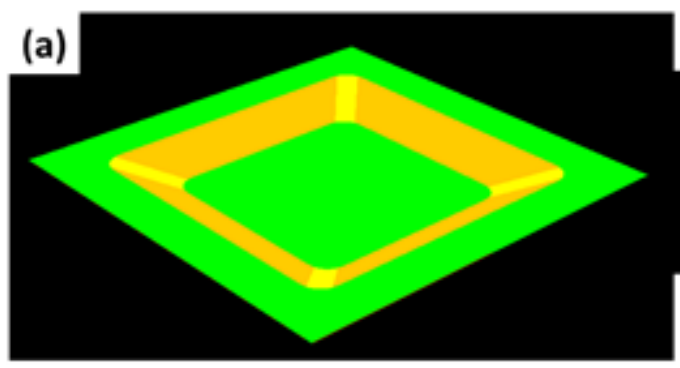

(b)
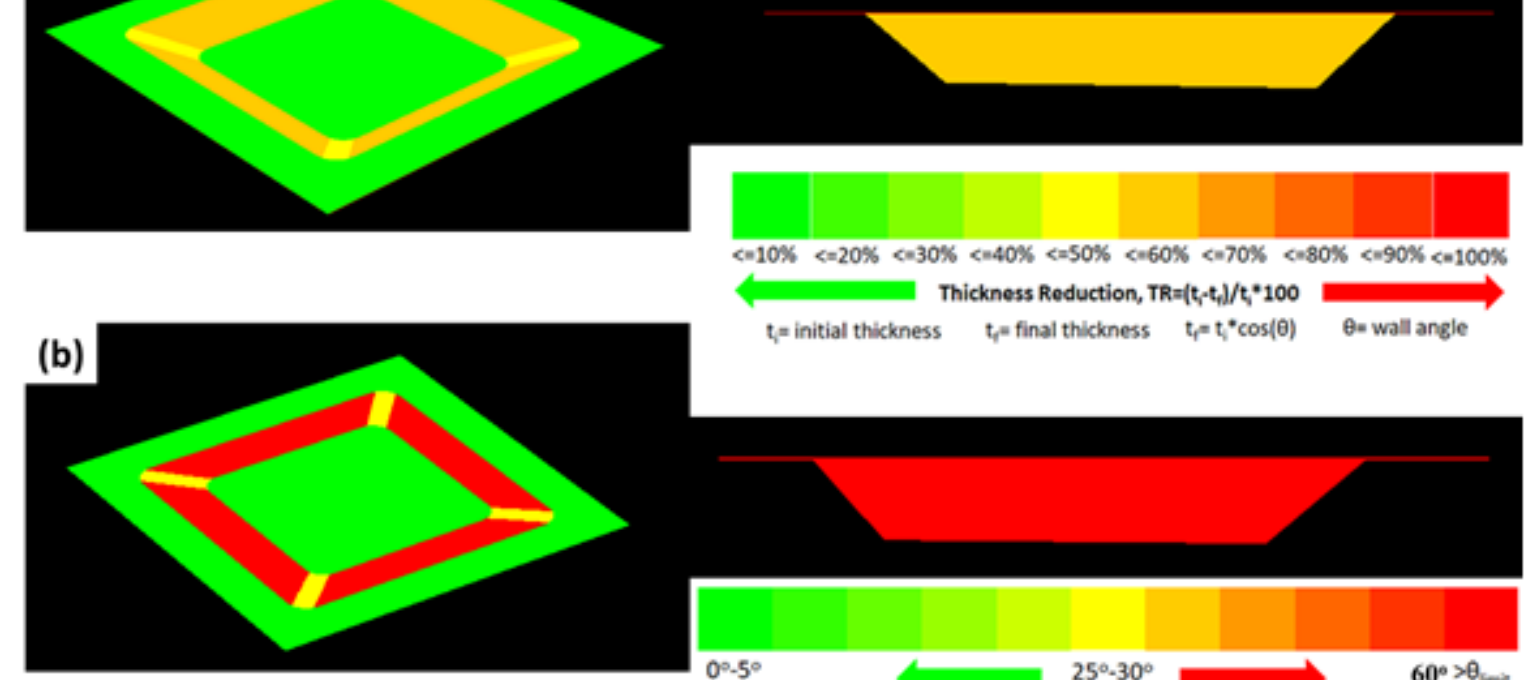

$t=$ initial thickness $\quad t=$ final thickness $\quad t, m t * \cos (\theta)$

$\theta=$ wall angle
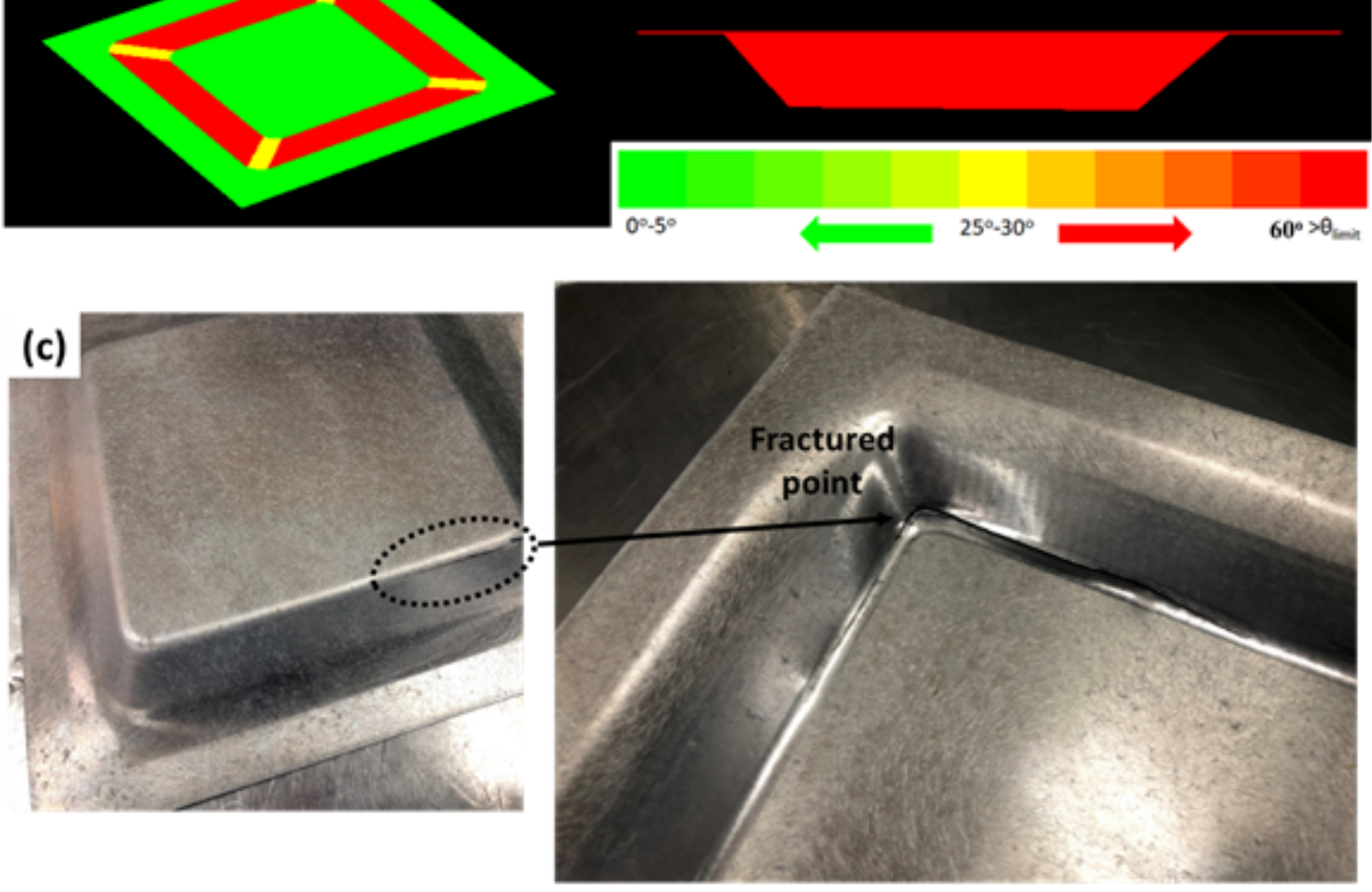

Figure 13

Truncated pyramid of 65 deg. wall angle (a) Part thinning (b) wall angle estimation using software (c) actual formed part 